\title{
Prognosis of motor development and joint hypermobility
}

\author{
E Tirosh, M Jaffe, R Marmur, Y Taub, Z Rosenberg
}

\begin{abstract}
In a study of 59 infants aged 18 months there were 20 with joint hypermobility and delayed motor development, 19 with joint hypermobility and normal motor development, and 20 normal controls. They were reassessed for motor function 3.5 years later at the age of 5 years. Both gross and fine motor performance were significantly delayed in the group of children who exhibited joint hypermobility and motor delay in infancy. No significant delay was evident in those with joint hypermobility only. Joint hypermobility resolved more frequently in children who presented normal motor development at age 18 months.

Infants with joint hypermobility and motor delay are a subgroup associated with a less favourable motor outcome and careful follow up is indicated.
\end{abstract}

The prevalence of joint hypermobility in infancy is about $17 \% .^{1}$ Previous reports have indicated an association between joint hypermobility and early motor delay, even when no evidence for a neurological deficit is demonstrated. ${ }^{12}$ It has been further suggested that this phenomenon is of a benign nature and that most infants with joint hypermobility will demonstrate a motor catch up by the age of 3 years. ${ }^{2}$

The aetiology of joint hypermobility, as well as the long term outcome of children with this entity, is unknown. Joint mobility can be determined by the structure of the joints and ligaments or by muscle tone. ${ }^{3}$ Muscle tone in turn may be associated with various abnormalities, including those in the central nervous system such as mental retardation or cerebral palsy. ${ }^{4}$ As fine motor function becomes increasingly sophisticated with age it was of particular interest to evaluate, in addition to gross motor achievement, the association between fine motor function and joint hypermobility in childhood.

The objective of the present study was twofold: (i) to assess prospectively the gross and fine motor proficiency of children who were identified as having joint hypermobility in infancy, either with or without motor delay, and (ii) to assess the association between joint hypermobility and motor function at the age of 5 years.

Subjects and methods

Sixty children between the ages of 54 and 60 months (mean 57.3 months) with no known neurological, genetic, or cognitive problems, were evaluated. Each group of 20 children was randomly selected out of the following categories diagnosed at the age of $\mathbf{1 8}$ months: group A, joint hypermobility with gross motor delay (20 out of the original group of 35 children); group B, joint hypermobility with normal motor development (20 out of the original group of 25); and group C, normal joints and normal motor development (20 out of the originally diagnosed 90).

The criteria for joint hypermobility were as previously described. ${ }^{1}$ After evaluation a skeletal deformity was found in one child in group B and he was excluded from the study. No significant differences in age $(p<0.3)$, sex $(p<0.5)$, or socioeconomic level $(p<0.4)$ were found between the three groups. Mean (SD) age at enrolment in nursery school was comparable $(24.8(10 \cdot 2)$ months) in the three groups $(\mathrm{p}<0 \cdot 14)$.

\section{PROCEDURE}

All children were evaluated by an experienced paediatric physiotherapist, who had not participated in the previous stages of the study and was not aware of the child's original diagnostic category. Ninety per cent reliability and $96 \%$ reproducibility of the joint assessment had been previously established. ${ }^{1}$ In addition, before the present evaluation, interobserver reliability of 1.0 for the joint evaluation and 0.9 for the gross motor measures was established among the examining physiotherapist, another physiotherapist, and a developmental paediatrician. No attempt was made to differentiate among the different mechanisms resulting in joint hypermobility.

\section{MEASURES}

Gross motor performance was evaluated with the Hoskins-Squires test for gross motor and reflex development. ${ }^{5}$ The 14 items which pertained to the age range of 36 to 60 months were administered and coded as pass/fail. A child who failed to perform at least $50 \%$ of the items was coded as 'fail', and conversely a success rate of $50 \%$ or more was coded as 'pass'.

Fine motor development and visual motor integration were assessed with the following three tests: (i) block tower (from the Miller assessment for the preschool child ${ }^{6}$ ), (ii) the Bruininks-Oseretsky pegboard tèst, ${ }^{7}$ and (iii) the Beery-Buktenica visual-motor integration test. $^{8}$

A measure of parental perception of motor 
proficiency was derived from nine items of a questionnaire designed specifically for the study (table 1). The Conners' parent rating scale was administered in order to rule out the possible contribution of short attention span and/or hyperactivity to inadequate performance on the motor tests.

One way analysis of variance and $\chi^{2}$ tests were used for analysis as appropriate.

\section{Results}

The prevalence of gross motor dysfunction at age 5 years among the children of group $A$ (joint hypermobility and motor delay in infancy) was significantly higher than in the other two groups $\left(\chi^{2}=19.83, p<0.003\right)$ (table 2). No significant difference was found between groups B and C. Those children who demonstrated joint hypermobility and motor delay at age 18 months were significantly more likely to present the same association when they reached the age of 5 years $\left(\chi^{2}=6.54, p<0.01\right)$ (table 2).

FINE MOTOR DEVELOPMENT AND VISUAL MOTOR INTEGRATION

Group A showed a significant disadvantage in the two fine motor tests. However, after Bonferoni correction, only the pegboard test remained significant. Forty five per cent of group A children failed the pegboard test, whereas only $21 \%$ of group B and 5\% of group C children failed this test $\left(\chi^{2}=7 \cdot 2, p<0.03\right)$. No difference in the visual motor integration test was found between the groups.

PARENTAL PERCEPTION OF MOTOR PROFICIENCY The parents of group A perceived their children as significantly less proficient in their motor abi-

Table 1 Child's motor proficiency: parental questionnaire

(1) Does your child play outdoors regularly with
other children?

(2) Circle which of the following activities are enjoyed by your child:

Swinging

Playing in the sand box

Sliding

Ball games

(3) Does your child prefer sitting in a push chair to walking?

(4) to walking? excessively?
ex your child

(5) Do you see your child as clumsy or with delayed motor development?

(6) Does your child trip or stumble a lot?

7) Does your child bump into objects or people excessively?

(8) Does your child wash his hands and dry them independently?

(9) Which of the following articles of clothing can your child put on independently? Shirt Trousers Socks lities than did the parents of the other two groups. Group A scored a mean (SD) of 2.5 (1.8), group B $1.1(1.6)$, and group C 0.7 $(0.9)$ on the parent's questionnaire $(F=8.0$, $\mathrm{p}<0.0008$ ).

No differences in perceived behaviour among the three groups were shown by the Conners' rating scale $(\mathrm{p}<0.83)$.

\section{Discussion}

The current prospective study is the first to provide data pertaining to motor ability outcome in children who presented with joint hypermobility in infancy. Previous reports addressed the associated gross motor patterns in infancy ${ }^{1}$ and early childhood. ${ }^{2}$ Those reports determined a gradual but complete resolution of the gross motor deficits. At the age of approximately 5 years it is possible to perform more sophisticated evaluations and thus diagnose more subtle dysfunction. It appears that 18 month old children with joint hypermobility and motor delay are at increased risk not only for gross motor, but also for fine motor problems in later childhood.

No statistical difference was found between the motor functions of children who were originally diagnosed as having joint hypermobility only and the normal controls. However, the former group scored consistently lower on the fine motor items.

At 5 years of age, the joint hypermobility of infants who had no motor delay at 18 months of age resolved more frequently (84\%) than that of infants whose joint hypermobility was associated with motor delay (40\%) (table 2 ).

The pathogenesis of this entity remains unknown. The fine motor findings at the age of 5 years suggest possible factors other than laxity of the ligaments and joint capsule or hypotonic muscle, as the fine motor function is not expected to be affected by these mechanisms. The course of this phenomenon varies, and infants with joint hypermobility but not motor delay at the age of 18 months seem to have a favourable outcome, whereas those with motor delay are at risk for both gross and fine motor problems in later childhood. It is therefore possible that there are two subsets of children with joint hypermobility, one group with normal motor development and no residual motor dysfunction and the other group with joint hypermobility and gross and fine motor dysfunction which may have its origin in the central nervous system. This subgroup might therefore benefit from a prolonged neurodevelopmental follow up. Whether their school performance will be affected remains to be investigated.

Table 2 foint and development status of children at 18 months and 5 years of age. Figures are given as number (\%)

\begin{tabular}{|c|c|c|c|c|c|}
\hline \multirow[t]{2}{*}{18 Months (group) } & \multicolumn{4}{|l|}{5 Years } & \multirow[t]{2}{*}{ Total } \\
\hline & $\begin{array}{l}\text { foint hypermobility } \\
\text { and motor delay }\end{array}$ & $\begin{array}{l}\text { Joint hypermobility } \\
\text { only }\end{array}$ & Motor delay only & Normals & \\
\hline $\begin{array}{l}\text { Joint hypermobility } \\
\text { and motor delay (A) } \\
\text { Joint. hypermobility only (B) } \\
\text { Normals (C) }\end{array}$ & $\begin{array}{l}8 \text { (40) } \\
1 \text { (5) } \\
1 \text { (5) }\end{array}$ & $\begin{array}{l}4(20) \\
2(11) \\
1(5)\end{array}$ & $\begin{array}{l}5(25) \\
4(21) \\
3 \text { (15) }\end{array}$ & $\begin{array}{r}3(15) \\
12(63) \\
15(75)\end{array}$ & $\begin{array}{l}20(100) \\
19(100) \\
20(100)\end{array}$ \\
\hline
\end{tabular}


We would like to thank the parents who participated in the study and Mrs Miriam Amit and Miss Ruth Singer for their valuable help in the preparation of the manuscript.

1 Jaffe M, Tirosh E, Cohen A, Taub Y. Relationship between joint hypermobility and motor development in early life. Arch Dis Child 1988;63:153-61.

2 Benady S, Ivanans T. Hypermobile joints: a benign cause of transitory motor delay in infancy. Clin Pediatr (Phila) 1978; 17:790-6.

3 Dubowitz V, ed. The floppy infant. 2nd Ed. London: William Heinemann Medical Books, 1980:115-25. (Dubowitz V ed. Clinics in developmental medicine. Vol 76.)
4 Dubowitz V, ed. Muscle disorders in childhood. London: WB Saunders, 1978:223-31.

5 Hoskins TA, Squires JE. Developmental assessment: 'a test for gross motor and reflex development.' Phys Ther 1973, 53:117-25.

6 Miller LS. Assessment for the preschooler: manual. Littleton, Colorado: Foundation for Knowledge in Development, 1982.

7 Bruininks RH. Bruininks-Oseretsky pegboard test of motor proficiency $(41 / 2-141 / 2)$. Circle Pines, Minnesota: American Guidance Service, 1978.

8 Beery KE, Buktenica NA. Developmental test of visual-motor integration. Chicago: Follett, 1967.

9 Conners CK. Rating scales for use in drug studies with children. Psychopharmacol Bull 1972; Special issue:Pharmadren. Psychopharmacol Bull
cology with children:29-84.

More about haemolytic uraemic syndromes

Regular readers of this journal will be familiar with recent British work on the clinical features, epidemiology, and microbiology of these syndromes (Archives of Disease in Childhood 1990:713-27) and the association with verocytotoxin producing Escherichia coli. A recent study from Buenos Aires has shown high rates of asymptomatic carriage in the parents and siblings of affected children. ${ }^{1}$ Verocytotoxin was found in the stools of $39 \%$ (25 of 64 ) of household contacts though $E$ coli 0157: $\mathrm{H7}$, the villain of the piece in the British studies, was not grown. Of the 24 contacts from whom paired sera were collected $42 \%$ (10) showed seroconversion for neutralising antibody to verocytotoxin.

In Salt Lake City 61 patients were examined five to 18 years after an episode of haemolytic uraemic syndrome. ${ }^{2}$ Nearly $\mathbf{4 0 \%}$ (24) had proteinuria, reduced creatinine clearance, or both, and three of these 24 were also hypertensive. The abnormalities sometimes appeared after a period of apparent normality. These authors therefore recommend long term follow up of all patients after an episode of haemolytic uraemic syndrome even for mild cases apparently recovered.

This recommendation is supported by members of the nephrology team at the Children's Hospital, Birmingham. ${ }^{3}$ They have shown that in children with a good outcome the urine ratio of protein to creatinine steadily declined over the first year to less than $20 \mathrm{mg} / \mathrm{mmol}$ in $87 \%$ of cases, whereas in those with a poor outcome the ratio invariably remained high.

Children who have apparently recovered from haemolytic uraemic syndrome should have a check on their blood pressure and an early morning urine protein:creatinine ratio from time to time throughout childhood and adolescence. Those with a high protein:creatinine ratio should be followed up more carefully. It is, of course, essential, if you haven't already done so, to persuade your laboratory to use a method capable of measuring low urine protein concentrations. The method used in Birmingham is the Coomassie blue dye binding technique.

1 Lopez EL, Diaz M, Devoto S, et al. Evidence of infection with organisms producing shiga-like toxins in household contacts of children with the hemolytic uremic syndrome. Pediatr Infect Dis $\mathcal{f}$ 1991;10:20-4.

2 Siegler RL, Milligan MK, Burnigham TH. Long-term outcome and prognostic indicators in the hemolytic-uremic syndrome. $\mathcal{f}$ Pediatr 1991;118:195-200.

3 Milford DV, White RHR, Taylor CM. Prognostic significance of proteinuria one yea after onset of diarrhoea-associated hemolytic-uremic syndrome. F Pediatr 1991;118: $191-4$. 